\author{
Military Technical College \\ Kobry Elkobbah, \\ Cairo, Egypt
}

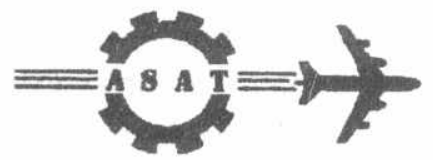

\author{
$8^{\text {th }}$ International Conference \\ on Aerospace Sciences; \&. \\ Aviation Technology
}

\title{
STEADY SUPERSONIC FLOW OVER RIGHT CIRCULAR CONE
}

\author{
Sherif Fouad Ali*
}

\begin{abstract}
The supersonic flow over a cone is of great practical importance in applied aerodynamics; Nose cones of many high-speed missiles and projectiles are approximately conical, as are the nose regions of fuselages of most supersonic airplanes. In the present work, a numerical technique for solving the Taylor-and-Maccoll conical flow equation is introduced. Results of the present technique are verified against results of other key researchers. An excellent agrecment was found in all cases. The inputs of the present code are the free stream Mach number and the cone half angle. The output includes the velocity field, the pressure field, the shock wave angle, and the stream lines directions. The running time on a Pentium $75 \mathrm{Mhz}$ is around $2 \mathrm{CPU}$-seconds for each case.
\end{abstract}

\section{KEY WORDS}

Conical Flow, Numerical Techniques.

\section{INTRODUCTION}

When a cone at zero angle-of-attack is flown by a supersonic flow, the problem is axisymmetric. Moreover, it is proven that the flow properties are constant along a ray from the cone vertex. Thus, although the problem appears to be three dimensional, it is actually onedimensional. Accordingly, its governing equation is an ordinary differential equation (ODE) rather than the partial deferential equations common in the Aerodynamics era.

As mentioned before; the supersonic flow over cone is of great practical importance in applied aerodynamics; the nose cones of many high-speed missiles and projectiles are approximately conical, as are the nose regions of fuselages of most supersonic airplanes. Also, it can be used as a starting solution for bodies of revolution when the Mach angle is smaller than the body nose angie.

The first solution for the supersonic flow over a cone was obtained by Busemann in 1929 [1] long before supersonic flow became fashionable. This solution was essentially graphical, and illustrated some of the important physical phenomena. A few years later, in 1933, G.I.Taylor and J.W.Maccol [2] represented a numerical solution, which is a hallmark in the evolution of compressible flow. Since then, many researchers attacked the problem numerically to make tables for the supersonic flow around cones at zero angle of attack [1-3].

The governing equation of this problem is a second order ODE, which needs two boundary conditions for the system to be well posed. One of them is easy to figure out, the rigid body

Lecturer, Aeronautical Department, Military Tcchnical Collcge, Cairo, Egypt 
B.C.. But, the second one is not obvious. Actually, there are two choices. 1) To choose the tangential speed on the cone surface, then get the corresponding shock angle and free stream Mach number as a result. 2) To choose the shock wave angle, then get the tangential speed on the cone surface and free stream Mach number as a result. In the present approach, there is no intention to make tables. But, the objective is to make an efficient numerical model and computer code that is capable of predicting the conical flow for the direct problem (given the free stream Mach number, and the cone semi-vertex angle solve the problem). Hence, integrating this code with another codes to solve real engineering problems. This is done by adopting the B.C. choice (1), and iterating to get the desired free stream Mach number. In another wards, the powerful computing facility, that is made available recently, is utilized to solve the direct problem. (The running time on a Pentium $75 \mathrm{Mhz}$ is around $2 \mathrm{CPU}$ seconds for each case.)

Results of the present technique are verified against key results [1, 5, and 6]. Excellent agreement was found in most cases.

\section{THEORETICAL BACKGROUND}

\subsection{GOVERNING EQUATIONS:}

The governing equation of the conical flow is obtained by applying the continuity equation, Euller's equation, and the adiabatic process thermodynamic relations in spherical coordinates (figs. 1 and 2). Also, the conical flow assumptions are used:

a) Axisymmitric flow $\frac{\partial}{\partial \varphi} \equiv 0$

b) Flow properties are constant along a ray from the cone vertex $\frac{\partial}{\partial} \equiv 0$

c) Irrotational flow $\nabla \times V \equiv 0$

The result is the Taylor-Maccoll equation for the solution of conical flow [5]

$$
\frac{\gamma-1}{2}\left[V_{\max }^{2}-V_{r}^{2}-\left(\frac{d V_{r}}{d \vartheta}\right)^{2}\right]\left[2 V_{r}+\frac{d V_{r}}{d \vartheta} \cot \vartheta+\frac{d^{2} V_{r}}{d \vartheta^{2}}\right]-\frac{d V_{r}}{d \vartheta}\left[V_{r} \frac{d V_{r}}{d \vartheta}+\frac{d V_{r}}{d \vartheta} \frac{d^{2} V_{r}}{d \vartheta^{2}}\right]=0
$$

Note that it is an ordinary differential equation, with only one dependent variable, $V_{r}$. Its solution gives $V_{r}=f(\vartheta)$.

The normal velocity can be calculated using the relation optioned from the continuity equation:

$$
V_{\vartheta}=\frac{d V_{r}}{d \vartheta}
$$

and the pressure field is evaluated using the relation[1]:

$$
\frac{p}{p_{03}}=\left[1-\left(\frac{V_{r}}{a}\right)^{2}-\left(\frac{V_{g}}{a}\right)^{2}\right]^{\gamma / \gamma-1}
$$




\subsection{BOUNDARY CONDITIONS:}

Equation (1) is a second order ODE that needs two boundary conditions for the mathematical model to be well posed.

a) Solid Cone Surface:

This is the common non-penetrable surface B.C., Which states that no flow is going through the surface:

$$
\text { When } \vartheta=\vartheta_{c} \quad V_{g}=\frac{d V_{v}}{d \vartheta}=0
$$

b) Shock Wave Condition:

By studding the shock polar, one can prove graphically a relation between the shock wave angle and the velocity vectors: [5]

$$
\text { When } \vartheta=\vartheta_{s} \frac{-1}{6} \frac{1-\left(\frac{V_{v}}{V_{\max }}\right)^{2}}{\left(\frac{V_{v}}{V_{\max }}\right)\left(\frac{V_{\vartheta}}{V_{\max }}\right)}=\tan \vartheta_{s}
$$

Equations ( 2 and 3 ) form a complete but inconvenient set of boundary conditions for the differential equation (1). A major difficulty being that we have no direct method for detecting the shock wave angle $\vartheta_{s}$ before solving the problem. Accordingly, some sort of iteration has to be used. Moreover, the differential equation (1) is nonlinear, second order, and with variable coefficients. That makes a closed analytic solution for that system is not an option. In the next section, a numerical technique for solving the above system is introduced and discussed.

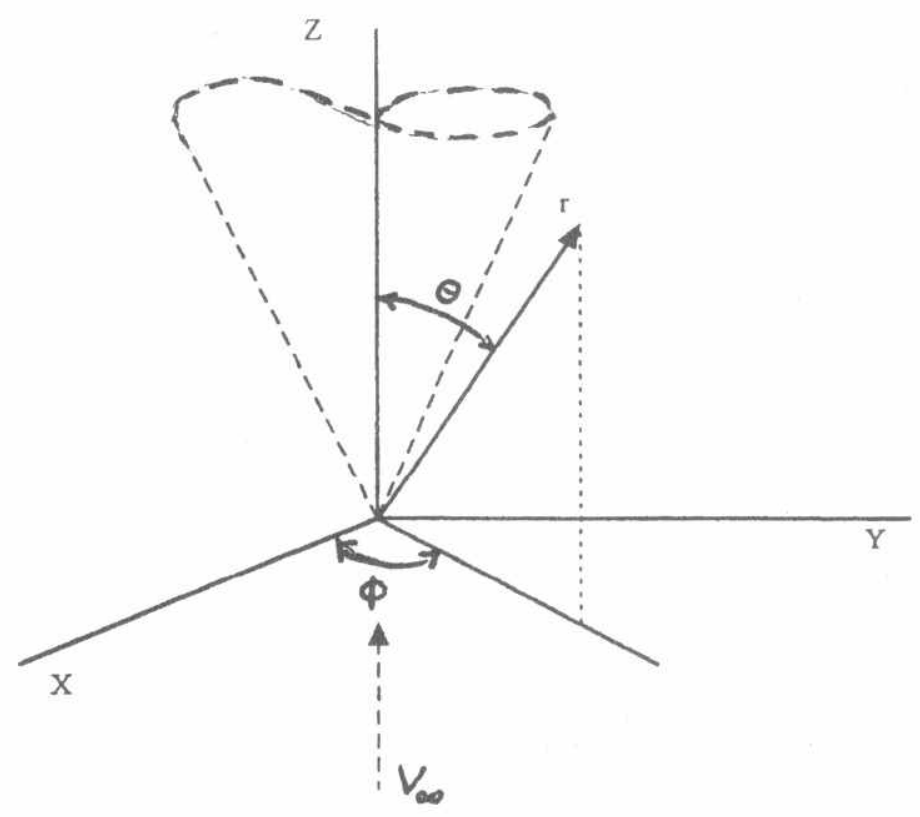

Figure 1.a: Spherical coordinate system 


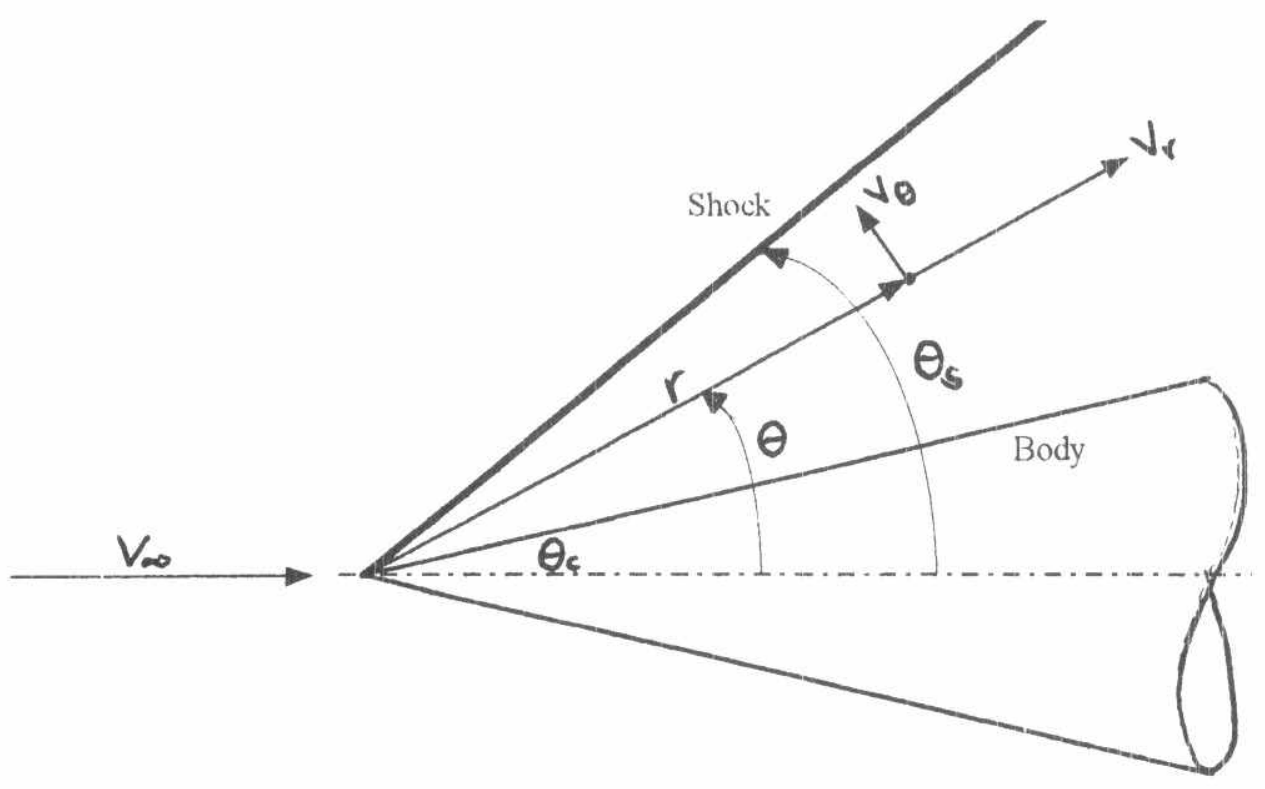

Figure 1.b: Spherical coordinate system for a cone

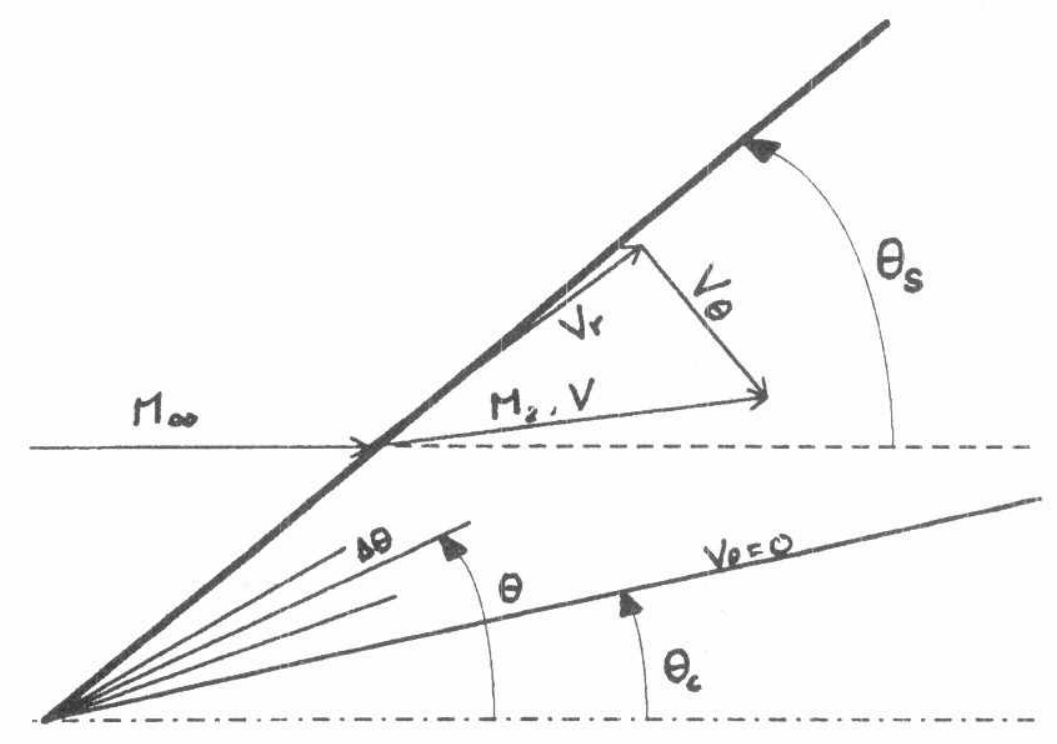

Figure 2: Geometry for the numerical solution of flow over a cone 


\section{NUMERICAL TECHNIQUE}

As mentioned in the previous section, equations 2 and 3 form a complete but inconvenient set of boundary conditions for equation 1. A major difficulty being that we have no direct method for determining the shock angle. Hence we cannot assign both $M_{\infty} \& \vartheta_{c}$ and get a direct solution.

Two methods for attacking this problem are possible:

1. The direct method (shock capturing), in which the cone semi-vertex angle is assigned, and the shock angle is obtained as a result.

2. The indirect method (shock fitting), in which the shock wave angle is assigned and the corresponding cone angle is obtained as a result.

The main objective of the present work is to make a convenient solution of the direct problem available and easy to use within a larger problem. Accordingly, the direct method is adopted in the present work.

\subsection{ODE INTEGRATION SUBROUTINE:}

A standard predicting-correcting technique for integrating a system of first-order ordinary differential equations is used [8]. A FORTRAN subroutine (ODE) is also available in the U.S. Government Library on the Internet. This subroutine requires the system of equations to be written in the form:

$$
\frac{d y(i)}{d t}=f(t, y(1), y(2), \ldots y(\text { neqns })) \text { and } \mathrm{i}=1 \text { to neqns }
$$

According,y, equation (1) is non-dimensionalised by $V_{\max }$ and rewritten in the form: (NOTE: Starting from here, all velocities are non-dimensionalised by $V_{\max }$ )

$$
\begin{aligned}
& y(1)=V_{r} \\
& y(2)=\frac{d V_{r}}{d \vartheta} \\
& y(3)=\frac{d^{2} V_{r}}{d \vartheta^{2}}=\frac{\left[2 y(1)\left(1-y(1)^{2}\right)+y(2)\left(1-y(1)^{2}\right) \cot \vartheta-7 y(1) y(2)^{2}-y(2)^{3} \cot \vartheta\right]}{\left(6 y(2)^{2}-1+y(1)^{2}\right)}
\end{aligned}
$$

Knowing $V_{r}$ at angle $\vartheta_{c}$ one can proceed to new angle with the desired accuracy.

\subsection{NUMERICAL PROCEDURE (suitable for computer programming):}

1- Read the free stream Mach number $M_{\infty}$, and the cone semi-vertex angle $\vartheta_{c}$.

2- Assume value for the radial velocity on the cone surface $V_{r}$ and solve (4) as IVP with steps $\Delta \vartheta$ moving away from the cone surface until (3) is satisfied. The obtained angle is the shock wave angle corresponds to the chosen $V_{r}$ on the surface. Also, calculate the corresponding Mach number after the shock wave $M_{2}$ using the following equation: 


$$
V=\sqrt{V_{r}^{2}+V_{g}^{2}}=\frac{1}{\sqrt{\frac{2}{(\gamma-1) M^{2}}+1}}
$$

3- Assume value for the radial velocity on the cone surface $V_{r}$ and solve (4) as IVP with steps $\Delta \vartheta$ moving away from the cone surface until (3) is satisfied. The obtained angle is the shock wave angle corresponds to the chosen $V_{r}$ on the surface. Also, calculate the conesponding Mach number after the shock wave $M_{2}$ using the following equation:

$$
V=\sqrt{V_{r}^{2}+V_{9}^{2}}=\frac{1}{\sqrt{\frac{2}{(\gamma-1) M^{2}}+1}}
$$

4-Usirig Oblique-Shock-Relations [6], calculate the free stream Mach number corresponding to $M_{2}$ and $\vartheta_{s}$ obtained from step 2 .

$$
\begin{aligned}
& M_{n 1}=M_{\infty} \sin \vartheta_{s} \\
& M_{n 2}=M_{2} \sin \vartheta_{s} \\
& M_{n 2}^{2}=\frac{M_{n 1}^{2}+\frac{2}{\gamma-1}}{\frac{2 \gamma}{\gamma-1} M_{n 1}^{2}-1}
\end{aligned}
$$

5-Compare the obtained Mach number with the required. Change the assumed value of the radial velocity on the cone surface $V_{r}$ and repeat 2-4 until you get the requirecl Mach number.

\section{RESULTS AND VALIDATION}

In order to check the accuracy and the efficiency of the present technique, various test cases are calculated and compared with well-known results of the present research problem.

5.1 The variation of pressure in the domain between the cone surface, and the shock wave:

In figure 3 , the pressure ratio obtained using the present technique is compared with that obtained by Taylor and Maccol [2]. For the shown results, the Mach number is $M_{\infty}=1.52$, and the cone semi-vertex angle is $\vartheta_{c}=30^{\circ}$. The horizontal axis is the angle in degrees measured from the cone centerline. The vertical axis is the static pressure divided by the total pressure after the shock The running time to get these results was around 2 CPU-seconds on a Pentium $75 \mathrm{MHz}$ computer. 


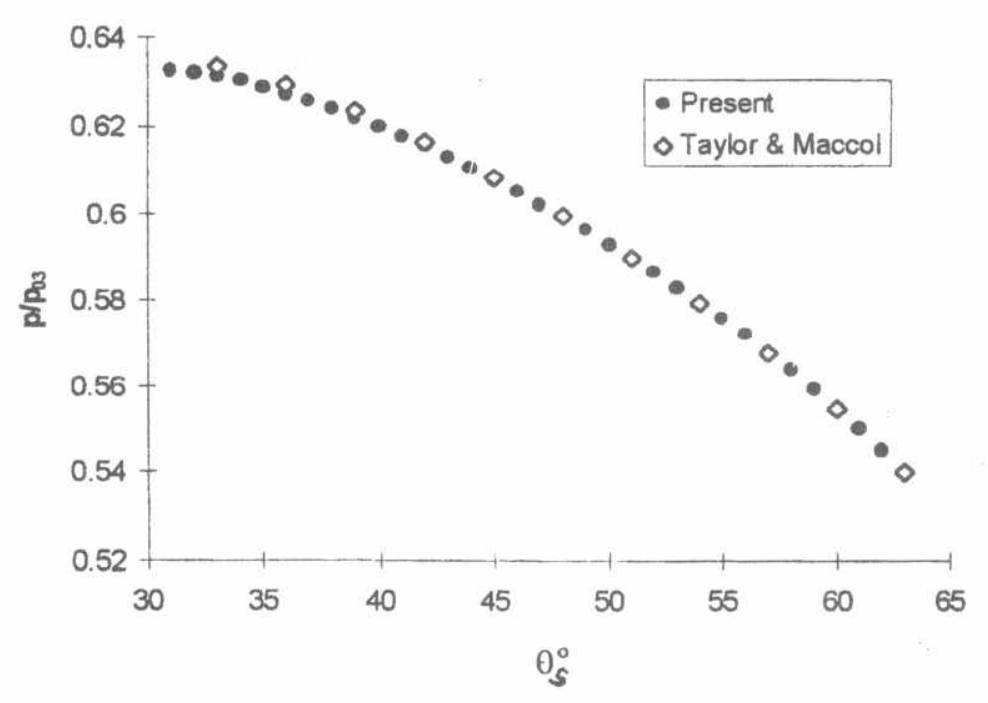

Figure 3: Pressure versus angle measured from the cone center line for $M_{\infty}=1.52$ and $\vartheta_{c}=30^{\circ}$

5.2 The variation of shock wave angle with Mach number:

In figure 4 , the variation of shock wave angle is drawn versus the free stream Mach number. Also, the cone angle appears in this figure as a parameter $\left(\vartheta_{c}=20^{\circ} \& 30^{\circ}\right)$. These results are obtained by choosing a fixed cone semi-vertex angle, then changing the free strearn Mach number to see how this will affect the shock wave angle [5]. Each point on this graph cost around 2 CPU-seconds. Hence, the total running time to obtain these results was about one CPU-minuet.

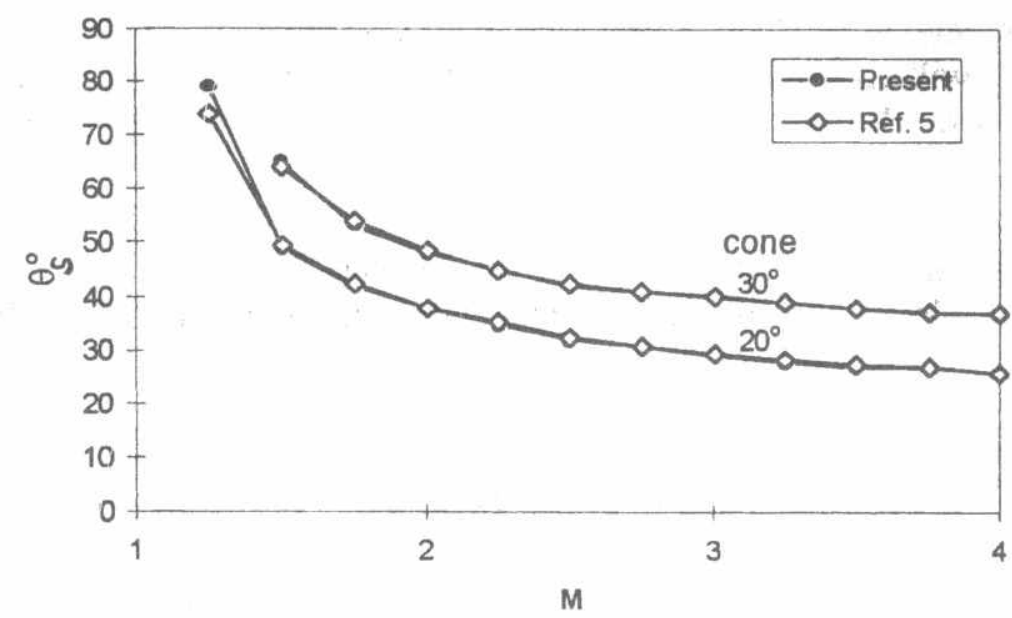

Figure 4: Shock wave angle versus free stream Mach number for $\vartheta_{c}=20^{\circ} \& 30^{\circ}$ 


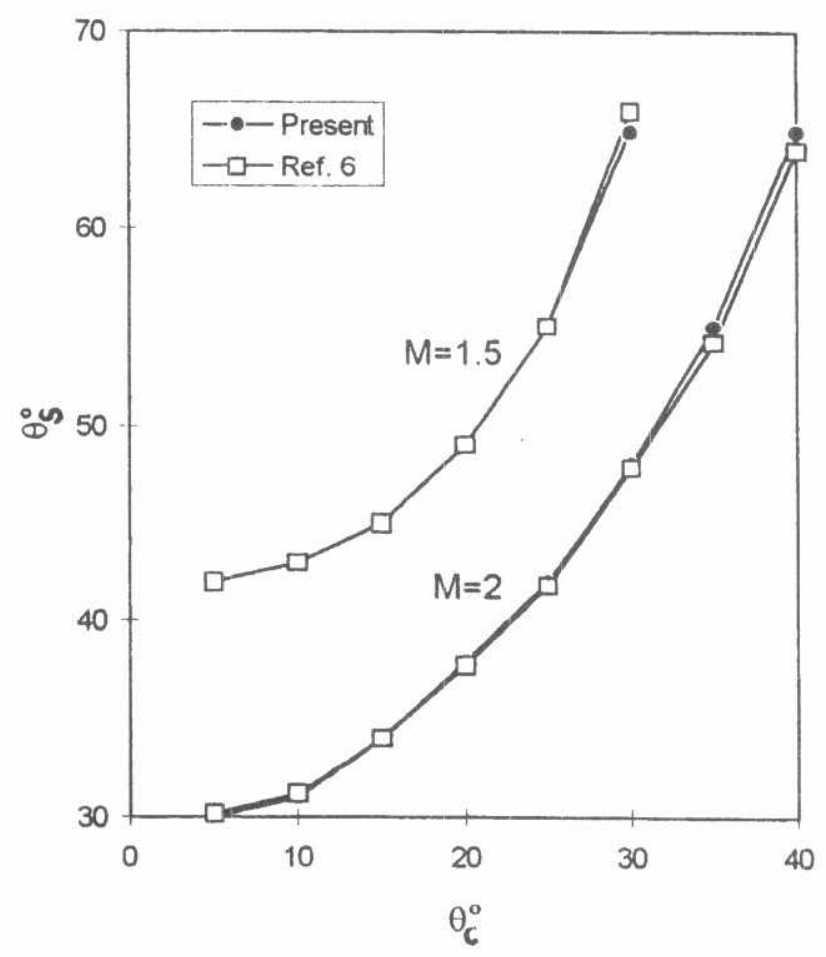

Figure 5: Shock wave angle versus cone semi-vertex angle for $M_{\infty}=1.5 \& 2$

\subsection{Variation of shock wave angle with the cone semi-vertex angle:}

In figure 5 , the variation of shock wave angle is drawn versus the cone semi-vertex angle. Also, the free stream Mach number appears in this figure as a parameter $\left(M_{\infty}=1.5 \& 2\right)$. These results are obtained by choosing a fixed free stream Mach number, then changing the cone semi-vertex angle to see how this will affect the shock wave angle [6]. Each point on this graph cost around 2 CPIJ-seconds Hence, the total running time to obtain these results was about one CPUminuet.

An excellent agreement was found in all test cases presented in this paper. The only exception is a convergence problem at high free stream Mach numbers, which will be studied in the future work.

\section{CONCLUSION}

An efficient numerical technique to solve the conical flow, based on Taylor and Maccol formulation, is introduced in this paper. Also, a FORTRAN computer code is built and tested. The results of the code are validated, and an excellent agreement was found.

The present technique solves the direct problem (given Mach number and cone angle, predict the flow parameters). This comes to the contrast of other techniques that solves the indirect problem (given the shock angle and the Mach number, predict the flow parameters). This makes the present technique more suitable to integrate with sophisticated problems (bodies of revolution, supersonic intakes, ...etc.).

The future work is to handle the angle of attack case, and the unsteady case. 


\section{REFERENCES}

1. A. Busemann, "Drucke auf kegelformige spitzen bei bewegung mit uberschallgeschwindigkeit,” Z. Angew Math. Mech., Vol. 9, 1929.

2. G.I. Taylor, and J.W. Maccoll, "The air pressure on a cone moving at high speeds," Proc. Roy. Soc. A, Vol.139, London, 1933.

3. Z. Kopal, "Tables of supersonic flow around cones," Technical report no.1, M.I.T., 19.47.

4. J.L. Smis, "Tables for supersonic flow around circular cones at zero angle-of-attack," NASA SP 3004, 1964.

5. E.R.C. Miles, "Supersonic Aerodynamics".

6. John D. Anderson, "Modern Compressible Flow, " McGraw-Hill Publishing Company, New York, 1990.

7. D. D. Liu, “Advanced Aerodynamics," MAE517 Lecture Notes, Arizona State University, 1994.

8. I.F. Shampine, and M.K. Gordon., "Computer solution of ordinary differential equations: The Initial Value Problem," FTP or e-mail: netlib@research.att.com. 